\title{
ARTHROPLASTY
}

\section{Low dislocation rates achieved when using dual mobility cup hip implants for femur neck fractures}

\author{
Erasmus $\mathrm{LJ}^{1} \mathbb{1}^{\mathrm{D}}$, Fourie $\mathrm{FF}^{2} \mathbb{1}$, Van der Merwe $\mathrm{JF}^{3}$ \\ 1 MBChB; Orthopaedic Surgery Registrar, University of the Free State, Universitas Academic Hospital, Bloemfontein, South Africa \\ 2 MBChB, MMed(Ortho), FC Orth(SA); Orthopaedic Surgeon, Life St Dominic's Hospital, East London, South Africa \\ 3 MBChB, MMed(Ortho), FC Orth(SA); Head of Arthroplasty Unit, University of the Free State, Universitas Academic Hospital, Bloemfontein, \\ South Africa
}

Corresponding author: Dr Lourens J Erasmus, Universitas Academic Hospital, 1 Logeman Street, Bloemfontein, Free State, 9301; PO Box 37140, Langenhovenpark, 9330; tel: +27 74149 8383; email: cobuserasmus@gmail.com

\begin{abstract}
Background: Total hip replacements done for intracapsular neck of femur fractures (NOF) have a dislocation rate of up to $14 \%$. This is seven times higher than in total hip arthroplasty (THA) done for osteoarthritis. Using a dual mobility cup (DMC) has been shown to be effective in addressing dislocation in elective THA. Our hypothesis is that the use of DMC in NOF will do the same. This study aims to determine the incidence proportion of dislocation of DMCs one year after surgery in patients who received THA for NOF and to compare it to dislocation rates as documented in existing studies.

Methods: A retrospective study was done on 86 patients treated with DMC THA for an intracapsular NOF fracture from 2012 until 2016. A minimum one-year follow-up period was required for inclusion into the study. The number of dislocations at one year after surgery was noted.

Results: Forty-one patients with a mean age of 60.7 years were included ( 26 females and 15 males). All patients were operated via the posterior approach. None of the patients had dislocated after one year.

Conclusion: Low dislocation rates can be achieved using DMC THA in the management of intracapsular NOF fractures. Our one-year dislocation rate of $0 \%$ compares favourably to conventional THA and is comparable to similar DMC studies done outside of South Africa.
\end{abstract}

Level of evidence: Level 4

Keywords: dual mobility cup, neck of femur fracture, dislocation, total hip arthroplasty, intracapsular

Citation: Erasmus LJ, Fourie FF, Van der Merwe JF. Low dislocation rates achieved when using dual mobility cup hip implants for femur neck fractures. SA Orthop J 2020;19(2):70-73. http://dx.doi.org/10.17159/2309-8309/2020/v19n2a2

Editor: Dr Michael Held, University of Cape Town, South Africa

Published: May 2020

Copyright: (c) 2020 Erasmus LJ. This is an open-access article distributed under the terms of the Creative Commons Attribution Licence, which permits unrestricted use, distribution and reproduction in any medium, provided the original author and source are credited.

Funding: No funding was received for this study.

Conflict of interest: The authors declare they have no conflicts of interest that are directly or indirectly related to the research. 


\section{Introduction}

The total hip replacement may have been rated as the best operation of the 20th century, but it is not without its complications. ${ }^{1}$ Dislocation of the hip prosthesis post-operatively remains one of the most common complications encountered after total hip arthroplasty (THA). Rates of $1.9 \%$ at one year and increasing up to $7 \%$ over 25 years have been reported in primary THA. ${ }^{2}$ Postoperative dislocations are the indication for surgery in $22.5 \%$ of revision cases and, even after revision surgery, $30 \%$ of patients will have persistent instability of their hip. ${ }^{3,4}$

The dislocation rate of THA done for fractures is much higher still, and thus of even more concern than in primary THA.

When using the posterior approach for THA done for femur neck fractures, Enocsen found a dislocation rate of $12-14 \% .{ }^{5}$ This is seven times higher than in primary hip arthroplasty. Hummel reported a dislocation rate of $2-8 \%$ when using the anterolateral approach for similar indications. ${ }^{6}$ If done for a failed open reduction and internal fixation of a femur neck fracture, $22 \%$ of hips dislocated post-operatively. ${ }^{7}$ When the patient was also demented, dislocation rates shot up to $32 \% .{ }^{7}$ As a local comparison, a study done at the University of Cape Town and published in 2018 found a 4.3\% risk for early dislocation after total hip arthroplasty for neck of femur (NOF) fractures. ${ }^{8}$

There are several patient risk factors that increase the risk for dislocation after THA. These include dementia, psychiatric disorders, alcohol abuse, age higher than 80 years old, neuromuscular disorders and non-compliance with post-operative movement and rehabilitation instructions. ${ }^{9,10}$

Besides patient risk factors, there are also surgical risk factors that contribute to dislocation. Some of these are the surgical approach used, the positioning of the acetabular and femoral components, soft-tissue tension and the surgeon's experience. ${ }^{9}$ Great emphasis was previously placed on putting the implant in the so-called 'safe zone' with the acetabular cup at $40^{\circ} \pm 10^{\circ}$ inclination and $15^{\circ} \pm 10^{\circ}$ anteversion. ${ }^{11}$ This has recently been found not to be as much of a protective factor as previously thought, with $58 \%$ of all hip prosthesis dislocations being in the safe zone. Abdel, who headed the study, concluded that hip dislocations post THA are multifactorial in cause, and a holistic approach needs to be taken to minimise the risk of dislocation. ${ }^{12}$

A modern solution to the problem of dislocation after THA has been suggested, namely the dual mobility cup (DMC). Designed by Prof. Gilles Bousquet and André Rambert in France in 1974, it features two articulations: the acetabular cup with the polyethylene insert and the polyethylene insert with the head of the femoral component. ${ }^{13-15}$ It is available in both cemented and uncemented options. This implant has been suggested as an option to reduce dislocation rates in very high-risk patients.

DMCs theoretically decrease dislocation risk for the same reasons a large effective femoral head does. It increases the head-to-neck ratio, allowing for a greater range of motion before impingement starts taking place. ${ }^{16,17}$ It also increases jump distance, allowing for a greater amount of lateral head movement before dislocation takes place. ${ }^{17}$

Some authors recommend caution when using it for standard primary THA and in young patients as there is some concern about increased wear rates and aseptic loosening. This concern is mainly because of the lack of long-term follow-up data and not because high rates of wear or aseptic loosening have actually been found..$^{13}$ There may in fact be decreased wear with DMCs as there are two articulating interfaces and thus less friction and sliding at each. ${ }^{18,19}$ Regarding real-world outcomes measured thus far, medium-term follow-up of these prostheses has been done by Philippot, who found a very favourable 15-year socket survival rate of $96.3 \%$ $( \pm 3.7 \%) .{ }^{20}$ Several companies currently offer DMC options for THA. Some examples are shown in Table $I$.

This study aims to determine whether DMCs used in NOF fractures are a possible solution to the high dislocation rates mentioned above. It intends to do so by retrospectively determining the cumulative incidence of dislocation in our study group at one year post-surgery and then comparing the numbers found to existing studies of dislocations in conventional total hip replacements as well as DMC studies done in other countries.

\section{Methods}

We did a retrospective cohort study at a single tertiary level hospital in Bloemfontein (Universitas Academic Hospital). We identified all the patients who had received DMC hip arthroplasty for intracapsular NOF fractures from July 2012 until December 2016. A total of 86 patients were identified. Electronic records (Meditech) and admission data, clinic files, radiological records and telephonic follow-up were then used to determine whether these patients had dislocated their hips post-operatively. Surgeon experience and the method of implant fixation were also documented.

A minimum follow-up time of one year (at our clinic or telephonic) was required to be included in the study. Patients who did not complete a full year of follow-up at our clinic were phoned to find out whether they had dislocated or not. Patients with incomplete records were excluded from the study. Those who had less than one year of follow-up time and were untraceable telephonically or otherwise were excluded. The Department of Home Affairs assisted in identifying patients who passed away within the first year of surgery. These patients were also excluded. Those who had arthroplasty done for failed open reduction and internal fixation of intracapsular NOF fractures were also excluded. Age was not an exclusion criterion. Some younger patients received DMC hip arthroplasty because of a high risk for dislocation. This decision was at the discretion of the attending consultant.

After determining the cumulative incidence of dislocations in our study group, we planned to compare our numbers to those of existing studies on hip dislocation in standard and DMC hip arthroplasty done for intracapsular NOF fractures.

All patients had a primary hip arthroplasty with a Polarcup ${ }^{\circledR}$ prosthesis. This product is manufactured by Smith \& Nephew

Table I: Dual mobility cups available

\begin{tabular}{|l|l|l|l|c|}
\hline Company & Trade name & HXPE* & Cemented & Head sizes (mm) \\
\hline Smith \& Nephew & Polarcup & Yes & Yes & 22,28 \\
\hline Tornier & Dual Mobility Cup & Yes & Yes & 22,28 \\
\hline Stryker & Mobile Hip System & Yes & Yes & 22,28 \\
\hline Zimmer-Biomet & Avantage & Yes & Yes & 22,28 \\
& Active Articulation & Yes & No & 28 \\
\hline DePuy & Gyros & Yes & No & $22.5,28$ \\
\hline
\end{tabular}

*HXPE: highly cross-linked polyethylene 
Orthopaedics AG of Rotkreuz, Switzerland. Several other companies also manufacture similar prostheses and the choice of this specific implant was based on departmental protocol. Both cemented and uncemented techniques were used for acetabular cups and femoral components, depending on patient indications. All the patients were operated via the posterior approach (KocherLangenbeck approach). This is departmental protocol and makes comparison with other DMC studies much easier and more accurate, as the vast majority (>95\%) of similar studies done previously also utilised the posterior approach.

\section{Results}

Forty-one of the 86 patients identified were included in the study. A total of 45 patients were excluded. Eight had incomplete files, four passed away during the first year after surgery and the rest did not complete a full year of follow-up and could not be contacted telephonically. Thirty-four of these patients followed up at our clinic for one year or more. Seven patients did not complete a full year of follow-up at the clinic but were reached telephonically more than one year after surgery was performed and were confirmed not to have dislocated.

The mean age of the patients included was 60.7 years (SD 8.6). Twenty-six (63.4\%) of the patients were females, with the youngest being 42 years, the oldest 81 years and with a mean age of 62 years (SD 9.5). There were $15(36.6 \%)$ males among the patients included, with the youngest being 49 , the oldest 67 and with a mean age of 58.4 years (SD 6.3). Patient demographics are shown in Table II.

The risk factors for dislocation common to all the patients in the cohort were that they received THA for a NOF fracture via the posterior approach. Surgeon experience could not be controlled for and prostheses fixation was variable (according to patient indications). Regarding the experience of the surgeon, 24 cases $(58.5 \%)$ were performed by a registrar, 12 cases $(29.3 \%)$ by a registrar with consultant supervision and five (12.2\%) by a consultant. Both cemented and uncemented prostheses were used in different combinations depending on specific patient indications. A cemented cup and stem was used in 29 of the cases (70.7\%). An uncemented cup and cemented stem (hybrid implant) was used in six patients (14.6\%). A cemented cup and uncemented stem

Table II: Patient demographics

\begin{tabular}{|l|c|}
\multicolumn{2}{|l|}{ Characteristics $(n=41)$} \\
\hline Age in years (mean; SD) & $60.7 ; 8.6$ \\
\hline Females & $62 ; 9.5$ \\
\hline Males & $58.4 ; 6.3$ \\
\hline Sex $(n ; \%)$ & \\
\hline Females & $26 ; 63.4 \%$ \\
\hline Males & $15 ; 36.6 \%$ \\
\hline
\end{tabular}

(reverse hybrid implant) was used in four of the cases (9.8\%) and an uncemented cup and stem was used in two patients (4.9\%). Details of the surgeries performed are summarised in Table III. Some complications other than dislocation were encountered. Two of the patients developed deep wound infections. One of these patients ended up having a Girdlestone excisional arthroplasty and the other had to undergo two-stage revision surgery.

The main aim of this study was to determine the cumulative incidence of dislocation of DMC hip prostheses used for intracapsular NOF fractures one year after surgery was performed. We found that none $(n=0)$ of the patients included in our study had dislocated one year after surgery.

\section{Discussion}

By using DMC THA in the management of intracapsular NOF fractures we achieved a $0 \%$ dislocation rate at one-year followup. This is significantly better than the rates reported with conventional THA for this indication. ${ }^{5,7,8}$ (This is compared to total hip replacements for NOF fractures in general, and not for specific prostheses like big femoral head components that might compare more favourably with DMC implants. $)^{21}$ The dislocation rates for DMCs found in this study are similar to the results found by other authors in recent years. Table $I V$ shows a comparison of the results of similar studies done. All the studies shown in Table IV used the posterior approach, except for the study done by Adam et al. in which $20 \%$ of cases were performed via the anterolateral approach. ${ }^{22}$

A limiting factor to this study is the large number of patients lost to follow-up. Universitas Academic Hospital has a catchment area that includes the Free State, Northern Cape, Lesotho and parts of the Eastern Cape. Many of these areas are very remote and

Table III: Details of surgeries performed

\begin{tabular}{|l|c|}
\hline Surgeries performed $(\mathbf{n = 4 1 )}$ & \\
\hline Prosthesis & $n(\%)$ \\
\hline Smith \& Nephew Polarcup & $41(100 \%)$ \\
\hline Approach & \\
\hline Posterior (Kocher-Langenbeck) & $41(100 \%)$ \\
\hline Surgeon experience & \\
\hline Registrar & $27(58.5 \%)$ \\
\hline Registrar with consultant supervision & $12(29.3 \%)$ \\
\hline Consultant & $5(12.2 \%)$ \\
\hline Fixation method & \\
\hline Cemented cup and stem & $29(70.7 \%)$ \\
\hline Uncemented cup and cemented stem & $6(14.6 \%)$ \\
\hline Cemented cup and uncemented stem & $4(9.8 \%)$ \\
\hline Uncemented cup and stem & $2(4.9 \%)$ \\
\hline
\end{tabular}

Table IV: Dislocation rates of dual mobility cups used for neck of femur fractures

\begin{tabular}{|l|c|c|c|c|c|c|}
\hline Authors & Year & Country & Follow-up & Number of patients $(\mathbf{n})$ & Dislocations $(\mathbf{n})$ & Dislocation rate (\%) \\
\hline Current study & 2019 & South Africa & 12 months & 41 & 0 & $0 \%$ \\
\hline Tarasevicius et al. ${ }^{23}$ & 2010 & Lithuania & 12 months & 42 & 0 & $0 \%$ \\
\hline Adam et al. ${ }^{22}$ & 2012 & France & 9 months & 214 & 3 & $1.4 \%$ \\
\hline Bensen et al. ${ }^{24}$ & 2014 & Denmark & 21 months & 175 & 8 & $4.6 \%$ \\
\hline Nich et al. ${ }^{25}$ & 2016 & France & 36 months & 45 & 3 & $3.7 \%$ \\
\hline Boukebous et al. ${ }^{26}$ & 2018 & France & 24 months & 98 & $3.1 \%$ \\
\hline Zagorov et al. ${ }^{27}$ & 2018 & Bulgaria & 29 months & 49 & 0 & $0 \%$ \\
\hline Rashed et al. ${ }^{28}$ & 2018 & Egypt & 12 months & 31 & 0 & $0 \%$ \\
\hline
\end{tabular}


rural which makes it difficult for patients to follow up in the long term. This is coupled with inadequate record-keeping, with many patients being admitted to hospital without having their telephone numbers or identity numbers captured. Despite excluding these patients from the study, we believe it is unlikely that many, or even any, of them dislocated. The structure of the health system in the Free State is such that patients who dislocated would have to be referred to Universitas Academic Hospital for reduction and would likely have been picked up in this manner.

Future researchers may consider doing a prospective study in which they can better control data capture and possibly attain a higher level of long-term follow-up. A prospective study could also look at whether patients have other risk factors for dislocation besides the ones that the patients in our cohort shared, namely THA done via the posterior approach for NOF fractures.

\section{Conclusion}

The results obtained in this study were comparable to similar studies done abroad and show promise for the use of DMCs to achieve low dislocation rates in this high-risk group of patients.

\section{Ethics statement}

The author/s declare that this submission is in accordance with the principles laid down by the Responsible Research Publication Position Statements as developed at the 2nd World Conference on Research Integrity in Singapore, 2010.

Ethical clearance for the study was received from the University of the Free State Ethics Committee (HSREC 165/2016). Permission to use medical records was obtained from the Free State Department of Health. Formal consent was not required for this study.

\section{Declaration}

The authors declare authorship of this article and that they have followed sound scientific research practice. This research is original and does not transgress plagiarism policies.

\section{Author contributions}

LJE collected and analysed the data, and wrote and edited the manuscript. FF contributed to the protocol and management of patients. FJvdM proposed the study concept, supervised the study and managed patients.

\section{Acknowledgements}

We thank Mr FC van Rooyen of the Department of Biostatistics of the University of the Free State for his help in analysing the data used in this study.

\section{ORCID}

Erasmus LJ (D) https://orcid.org/0000-0003-4522-2873

Fourie FF (D) https://orcid.org/0000-0001-8961-4874

Van der Merwe JF (D) https://orcid.org/0000-0002-5554-4887

\section{References}

1. Learmonth ID, Young $\mathrm{C}$, Rorabeck $\mathrm{C}$. The operation of the century: total hip replacement. Lancet. 2007;370(9597):1508-19.

2. Berry DJ, Von Knoch $M$, Schleck CD, Harmsen WS. Effect of femoral head diameter and operative approach on risk of dislocation after primary total hip arthroplasty. J Bone Joint Surg Am. 2005;87(11):2456.

3. Bozic KJ, Kurtz SM, Lau E, et al. The epidemiology of revision total hip arthroplasty in the United States. J Bone Joint Surg Am. 2009;91(1):128-33.

4. Woo RY, Morrey BF. Dislocations after total hip arthroplasty. J Bone Joint Surg Am. 1982;64(9):1295-306.

5. Enocson A, Hedbeck C, Tidermark J, et al. Dislocation of total hip replacement in patients with fractures of the femoral neck. Acta Orthop. 2009;80(2):184-89.

6. Hummel MT, Malkani AL, Yakkanti MR, Baker DL. Decreased dislocation after revision total hip arthroplasty using larger femora head size and posterior capsular repair. J Arthroplasty. 2009;24(6 Suppl):73-76.

7. Johansson T, Jacobsson SA, Ivarsson I, Knutsson A, Wahlström O. Internal fixation versus total hip arthroplasty in the treatment of displaced femoral neck fractures: a prospective randomized study of 100 hips. Acta Orthop. 2000;71(6):597-602.

8. Shituleni S, Maqungo S. Displaced intracapsular neck of femur fractures: Dislocation rate after total hip arthroplasty. SA Orthop J. 2018;17(1).

9. Dargel J, Oppermann J, Brüggemann GP, Eysel P. Dislocation following total hip replacement. Dtsch Arztebl Int 2014;111:884-90.

10. Woolson S, Rahimtoola Z. Risk factors for dislocation during the first 3 months after primary total hip replacement. $J$ Arthroplasty. 1999;14(6):662-68.

11. Lewinnek G, Lewis J, Tarr R, Compere C, Zimmerman J. Dislocations after total hip-replacement arthroplasties. $J$ Bone Joint Surg Am. 1978;60(2):217-20.

12. Abdel $M$, Von Roth $P$, Jennings $M$, Hanssen $A$, Pagnano $M$. What safe zone? The vast majority of dislocated THAs are within the Lewinnek safe zone for acetabular component position. Clin Orthop Relat Res. 2015;474(2):386-91.

13. Grazioli A, Ek E, Rüdiger H. Biomechanical concept and clinical outcome of dual mobility cups. Int Orthop. 2012;36(12):2411-18.

14. Ko L, Hozack W. The dual mobility cup. Bone Joint J. 2016;98-B (1 Suppl A):60-63.

15. De Martino I, Triantafyllopoulos GK, Sculco PK, Sculco TP. Dual mobility cups in total hip arthroplasty. World J Orthop. 2014;5(3):180-87.

16. Malik A, Maheshwari A, Dorr L. Impingement with total hip replacement. J Bone Joint Surg Am. 2007;89(8):1832-42.

17. Banerjee $\mathrm{S}$, Pivec $\mathrm{R}$, Issa $\mathrm{K}$, et al. Large-diameter femoral heads in total hip arthroplasty: an evidence-based review. Am J Orthop. 2014;43(10):506-12.

18. Stulberg S. Dual poly liner mobility optimizes wear and stability in THA: Affirms. Orthopedics. 2011;34(9):e445-e448.

19. Netter J, Hermida J, Chen P, Nevelos J, D'Lima D. Effect of microseparation and third-body particles on dual-mobility crosslinked hip liner wear. J Arthroplasty. 2014;29(9):1849-53.

20. Philippot R, Farizon F, Camilleri J, et al. Survival of cementless dual mobility socket with a mean 17 years follow-up. Chir Orthop Reparatrice Appar Mot. 2008;94(8):e23-e27.

21. Pituckanota $\mathrm{K}$, Arirachakaran $\mathrm{A}$, Tuchinda $\mathrm{H}$, et al. Risk of revision and dislocation in single, dual mobility and large femoral head total hip arthroplasty: systematic review and network meta-analysis. Eur J Orthop Surg Traumatol. 2018:28(3):445-55.

22. Adam $P$, Philippe $R$, Ehlinger $M$, et al. Dual mobility cups hip arthroplasty as a treatment for displaced fracture of the femoral neck in the elderly. A prospective, systematic, multicenter study with specific focus on postoperative dislocation. Orthop Traumatol Surg Res. 2012;98(3):296-300

23. Tarasevicius S, Busevicius $M$, Robertsson $O$, Wingstrand $H$. Dual mobility cup reduces dislocation rate after arthroplasty for femoral neck fracture. BMC Musculoskelet Disord. 2010;11(1).

24. Bensen A, Jakobsen T, Krarup N. Dual mobility cup reduces dislocation and re-operation when used to treat displaced femoral neck fractures. Int Orthop. 2014;38(6):1241-45.

25. Nich C, Vandenbussche E, Augereau B, Sadaka J. Do dual-mobility cups reduce the risk of dislocation in total hip arthroplasty for fractured neck of femur in patients aged older than 75 years? J Arthroplasty. 2016;31(6):1256-60.

26. Boukebous B, Boutroux P, Zahi R, Azmy C, Guillon P. Comparison of dual mobility total hip arthroplasty and bipolar arthroplasty for femoral neck fractures: A retrospective case-control study of 199 hips. Orthop Traumatol Surg Res. 2018;104(3):369-75.

27. Zagorov M, Mihov K, Dobrilov S, et al. Dual mobility cups reduce dislocation rate in total hip arthroplasty for displaced femoral neck fractures. J of IMAB. 2018;24(2):2077-2081.

28. Rashed $R$, Sevenoaks $H$, Shabaan $A$, et al. Functional outcome and health related quality of life after dual mobility cup total hip replacement for displaced femoral neck fractures in middle aged Egyptian patients. Injury. 2018;49(3):667-72. 